\title{
Time runs out for space telescope
}

\section{Guidance snags} licked, others still hidden

\section{Washington}

Many fingers were crossed at a groundbreaking ceremony held at Johns Hopkins University in Baltimore, Maryland, on Monday to mark the beginning of construction work on the Space Telescope Science Institute. The construction of the Large Space Telescope itself, due to be launched from the space shuttle early in 1985 , seems just about on schedule and no major technical hurdles are foreseen.

Those who attended a programme review at the National Aeronautics and Space Administration (NASA)'s Marshall Space Flight Center two weeks ago, however, came away nervous that unanticipated problems could disrupt the delicate equilibrium in which the whole project seems to be balanced.

The Space Telescope Science Institute will be the principal observation and data analysis centre for the telescope, which will be placed in orbit 500 kilometres above the Earth's surface. It is being built on Johns Hopkins' Homewood Campus by the Association of Universities for Research in Astronomy under an initial five-year, \$24 million contract with NASA. Dr Riccardo Giacconi has been named director of the institute, which will eventually have a staff of about 150 , including between 30 and 40 astronomers from the United States and abroad.

The good news on the telescope is that the two mirrors which make up its RitcheyChrétien optical system are almost completed and are said to work beyond expectations. But there is less certainty about the eventual outcome of the instrumentation being developed to maintain the direction and stability of the telescope in orbit.

Initial designs for the fine-guidance sensor called for an image dissector able to capture a selected guide star combined with a fixed interferometer to compare the separate images of the star produced. When this proved to be a cumbersome arrangement Perkin-Elmer, the principal contractor for the Optical Telescope Assembly, put forward a more streamlined design which incorporated the moving parts into the interferometer. Once the start selector had found the guide star, the whole fine-guidance system would be locked in place, and directioning control transferred to the gyroscopes which are used to position the space support module within which the optical telescope assembly is contained. The scheme was calculated to operate with a high degree of accuracy.

Unfortunately it was realized at a late stage that, as the fine guidance sensor had a limited near range, by locking it in place the system would be unable to compensate for any sudden torque produced in the system as a whole in reaction to the movement of some other component if the result was a linear displacement of more than about 0.015 arc seconds.

Following this "pointing crisis", the whole system has been redesigned. New feedback has been introduced by keeping the star selector in the interferometer working in an active mode to keep the telescope fixed in its predetermined direction. Several parts of the five scientific

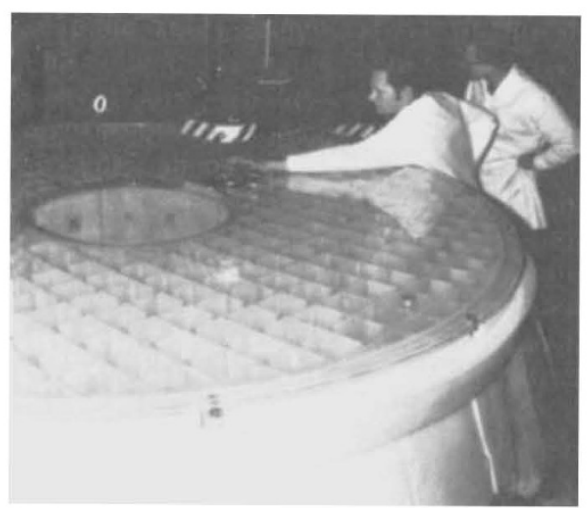

Primary mirror - looking good

instruments being carried are also being altered to reduce the effects of their movement on the rest of the vehicle.

However, the additional movement in the pointing system could still cause problems if either the fine-guidance sensor

\section{From the frying-pan into the fire?}

The British Prime Minister, Mrs Margaret Thatcher, announced earlier this week that the Secretary of State for Education and Science since May 1979, Mr Mark Carlisle, has been replaced by Sir Keith Joseph, Secretary of State for Industry during the same period. The changes are part of a substantial reorganization of the British Cabinet.

Mr Carlisle will now become an ordinary Member of Parliament. Sir Keith will remain a member of the Cabinet, in which he has been one of the principal advocates of monetarist policies since the election of the present government, but the post of Secretary of State for Education and Science is usually reckoned to carry with it less political clout than that at industry.

Even so, and in spite of Sir Keith's reputation for agonizing hard and long (and sometimes too long) before making a decision, the arrival of a new minister at the department will be welcomed in higher education. $\mathrm{Mr}$ Carlisle's uncompleted business includes the need to respond to the demand of the House of Commons Select Committee on Education for a sight of the or the gyroscopes create some unanticipated resonance in the support structure, something that will only be discovered during the pre-launch phase when a modal survey will be carried out.

The alterations will also mean a slight degradation in the telescope's expected performance. This will still be well within the minimum performance included in the original design specifications; but it will not, at least initially, be as high as it could have been if more time (and development funds) had been available.

Under the original Perkin-Elmer design, for example, the telescope was expected to be able to maintain an accuracy of 0.007 arc seconds over extended periods of time. The new system is designed to an accuracy of 0.012 arc seconds although it is hoped that in practice this can be raised to 0.009 arc seconds. Although much higher than most astronomers need, this will, for example, result in a loss of some image quality in the ultra violet range with the faint-image camera being built by the European Space Agency.

According to project scientist Dr Robert O'Dell, however, the main problems faced by the space telescope are not technical but financial. The development programme costing about $\$ 120$ million a year - is at the stage when delays can have the most significant impact on overall costs.

Late last year NASA reviewed the programme in view of problems that seemed to be emerging at Perkin-Elmer. This set the launch date back by eighteen months to early 1985 , and the total predicted cost was increased by about $\$ 150$ million in 1982 dollars to between $\$ 700$ and $\$ 750$ million.

letters written to him by the chairman of the University Grants committee, Dr Edward Parkes, and the need to respond to the demand by the Committee of ViceChancellors and Principals that funds should be made available to pay for the cost of redundancies among academics forced on the universities in July. The arrival at the Department of Education and Science of a secretary of state free to disown his predecessor's decisions will seem to many universities to be an opportunity for reprieve.

The reshuffle may, however, have come too late for some universities. Those which have already drawn up plans for reducing academic payrolls by compulsory redundancy may find that their compliance is taken as a sign of weakness. The rest are likely now argue that a slower contraction of the university system than decreed will in the long run be more economical.

The Space Telescope remains at the top of NASA's space science priority list. Proposed to Congress by President Carter in 1977 , it is the last "new start" to have escaped budget cuts, and its projected launch date has remained fixed on the 